\title{
Scleroderma renal crisis as the sole presenting feature of systemic sclerosis in a postpartum woman
}

\author{
Claudia Cobilinschi ${ }^{1,2}$, Cristian Cobilinschi ${ }^{1,3}$, Alexandra Constantinescu', \\ Ruxandra Ionescu ${ }^{1,2}$, Daniela Opris-Belinski ${ }^{1,2}$ \\ 1"Carol Davila" University of Medicine and Pharmacy, Bucharest, Romania \\ 2"Sf. Maria" Clinical Hospital, Bucharest, Romania \\ ${ }^{3}$ Intensive Care Unit, Clinical Emergency Hospital, Bucharest, Romania
}

\begin{abstract}
Systemic sclerosis is a chronic autoimmune disorder characterized by multiorgan involvement, most notably of the skin through fibrosis and vasculopathy. One of its most feared complications requiring rapid intervention is scleroderma renal crisis, as it can be fatal in the absence of prompt treatment. A 34-year old woman presents with a history of acute renal failure and malignant hypertension occurring one month postpartum and no other scleroderma feature in the following 5 years. Eventually, skin, heart and lung involvement is observed, positive anti-ARN III polymerase antibodies and suggestive capillaroscopic findings. Immunosuppressive therapy with mycophenolate mofetil is initiated and later switched to off-label Rituximab, with significant improvement of disease manifestations. Regular patient monitoring for novel symptom occurrence and appropriate treatment adjustment is essential for optimal management of scleroderma.
\end{abstract}

Keywords: systemic sclerosis, scleroderma renal crisis, ARN-polymerase III antibodies, postpartum, myocarditis, rituximab

\section{INTRODUCTION}

Systemic sclerosis (SSc) or scleroderma is a rare autoimmune connective tissue disease, its distinguishing feature being skin thickening mostly affecting the hands and face, with potential multisystem involvement, notably the lungs, heart, kidneys and gastrointestinal tract. The pathogenesis of SSc consists of inflammation, vascular injury and extensive interstitial fibrosis (1). It affects women four times more frequently than men and is characterized by a chronic, often progressive course, leading to significant morbidity and mortality. Based on the extent of skin involvement, systemic sclerosis can be broadly categorized into either limited or diffuse cutaneous variants, with differing patterns of disease progression (2).

One of the most daunting complications of this disorder is scleroderma renal crisis, manifested as acute kidney failure associated with hypertension, which if left untreated can become life-threatening. It occurs in approximately $5-10 \%$ of all systemic sclerosis patients but can affect up to $20 \%$ of individuals in the diffuse cutaneous disease subgroup. Anti-RNA polymerase III antibody positivity correlates with a higher risk of renal involvement, as it is present in approximately $50 \%$ of patients who develop this complication versus only $20 \%$ of overall systemic sclerosis patients. Corticosteroid therapy, particularly in high doses (over $15 \mathrm{mg}$ per day) or over a prolonged period of time, as well as cyclosporine use, rapidly-progressive skin involvement and tendon friction rubs are also considered important predictive factors (3) for developing renal complications.

It is notable that scleroderma renal crisis frequently occurs early in the course of the disease and can sometimes even be the presenting feature, preceding skin involvement. Up to half of the individuals who 

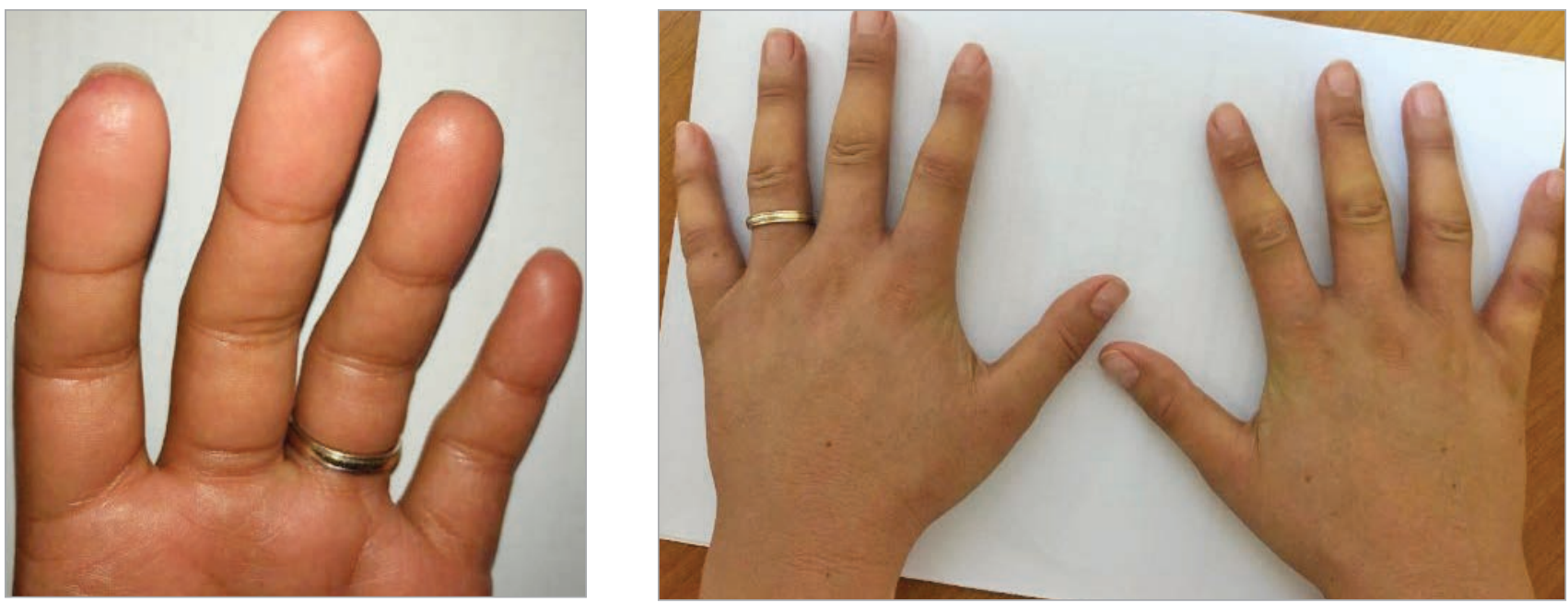

FIGURE 1. Pitting scars, sclerodactily and Raynaud's

develop this complication end up requiring dialysis, although angiotensin-converting enzyme inhibitor therapy has significantly improved the outcome for these patients (3).

\section{CASE PRESENTATION}

A 34-year old female presents with Raynaud's phenomenon, pain affecting the small joints of the hands, fingertip pitting scars and puffy fingers that occurred few months prior. History dating five years earlier reveals sudden onset of acute kidney failure, along with secondary malignant hypertension and acute pulmonary edema one month after giving birth. At the time, the patient was admitted in an ICU Department with progressive fatigue, headaches, high blood pressure, oligoanuria, nausea. Blood tests showed highly elevated creatinine and blood urea nitrogen levels, low serum albumin, nephritic-range proteinuria and moderately increased inflammatory markers. Echocardiography revealed low ejection fraction of $25 \%$, global hypokinesia, raising concerns of possible postpartum cardiomyopathy with heart failure. Kidney biopsy was performed and revealed mild glomerular injury, with interstitial inflammation and sclerosis, suggesting preeclampsia.

The patient was started on hemodialysis and hypotensive therapy with angiotensin-converting enzyme inhibitors, with renal function improving slowly over time, until dialysis was no longer required at five months.

Multiple laboratory tests were performed at the time, searching for other potential infectious or immunological conditions, however all antibody testing was negative, including anti SCL 70, anti centromere, anti U1 RNP, anti dsDNA, ACPA, anti-GBM, ANCA as well as cryoglobulins and antiphospholipid syndrome screening. Complement levels were within the normal range and nailfold capillaroscopy revealed no abnormalities. Suspicion of possible scleroderma was raised considering patient's description of Raynaud-like changes, despite no skin involvement at the time. The following years were free of any disease signs or symptoms, except for intermittent Raynaud'.

During the current rheumatology assessment, sclerodactyly and fine perioral radial furrowing are observed.

Capillaroscopy still shows no changes, yet anti-RNA polymerase III antibody titer show positive values.

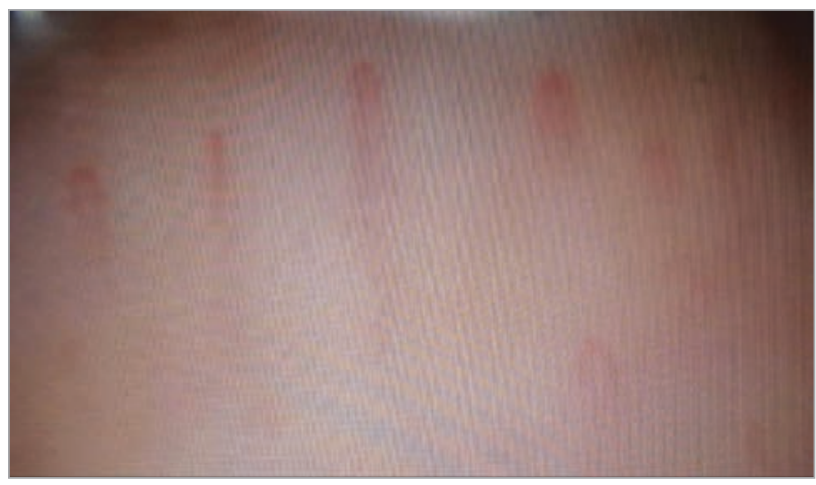

FIGURE 2. Nailfold capillaroscopy showing no megacapillaries, no hemorrhages

A further thoracic computed-tomography scan reveals basal ground-glass-like aspect, indicating moderate fibrosis, while high NT proBNP levels were identified and an additional cardiac MRI confirmed diffuse myocardial edema, with no fibrotic areas.

Considering Raynaud's phenomenon, discrete sclerodactily, pitting scars and antibody positivity, the patient is ultimately diagnosed with systemic sclerosis, according to the 2013 ACR/EULAR criteria with possible pulmonary and cardiac involvement. 


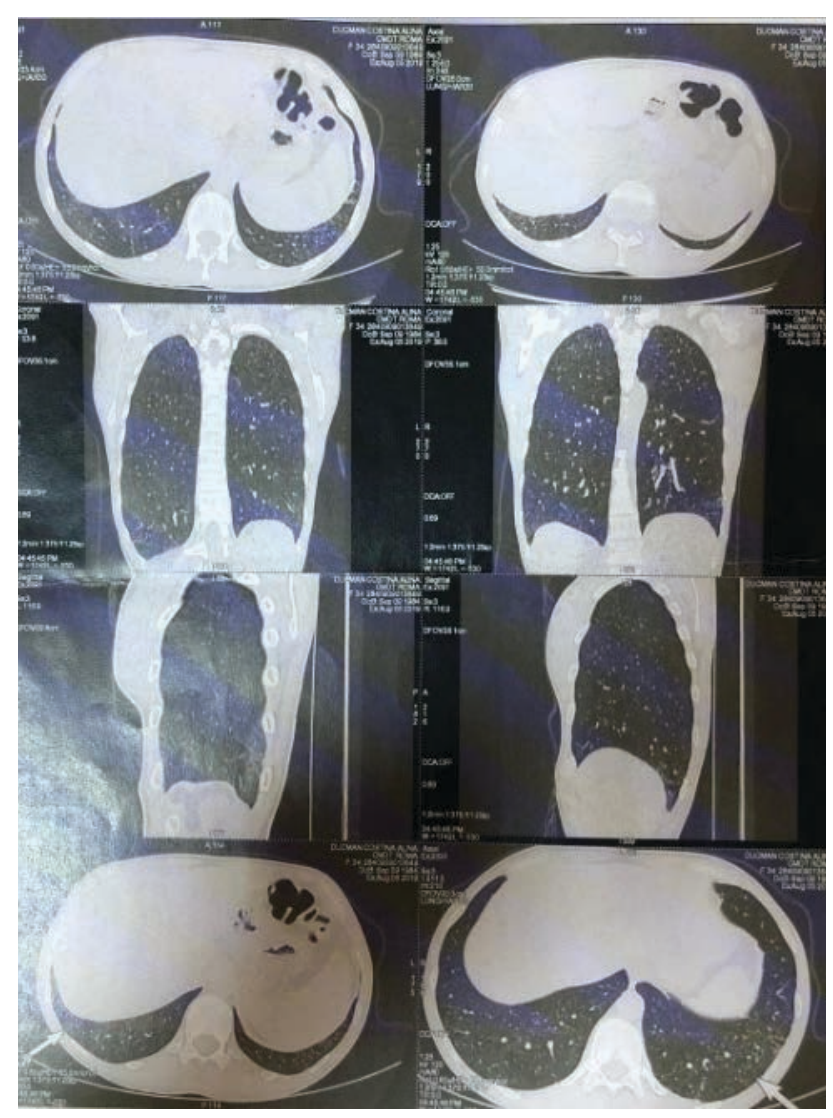

FIGURE 3. Thoracic CT showing fibrotic-like changes

Treatment is initiated with mycophenolate mofetil (MMF) in agreement with the patient, due to potential cardiotoxicity of cyclophosphamide and potential risk of worsening the pulmonary fibrosis with methotrexate. The treatment was well tolerated with progressive increase of MMF dose up to two grams.

However, at one-month evaluation, a significant skin change is noticed with progression of indurated areas that extended to the forearm, face anterior thorax and thighs, with a modified Rodnan score (mRSS) of 12. Moreover, facial telangiectasia are visible and accentuated pursed lips.

Nailfold capillaroscopy is once again repeated and reveals a typical active scleroderma pattern. Treatment options are reassessed and the decision to start Rituximab in addition to mycophenolate mofetil is made. At one month, therapy response is favorable and well tolerated, disease progression is halted and gradual skin loosening is observed. A repeated cardiac MRI shows no areas on inflammation in the myocardial muscle and the NT-proBNP measure is normal.

\section{DISCUSSIONS}

Scleroderma renal crisis is probably the most severe complication of systemic sclerosis, necessitating early recognition and prompt treatment initiation. Pri-

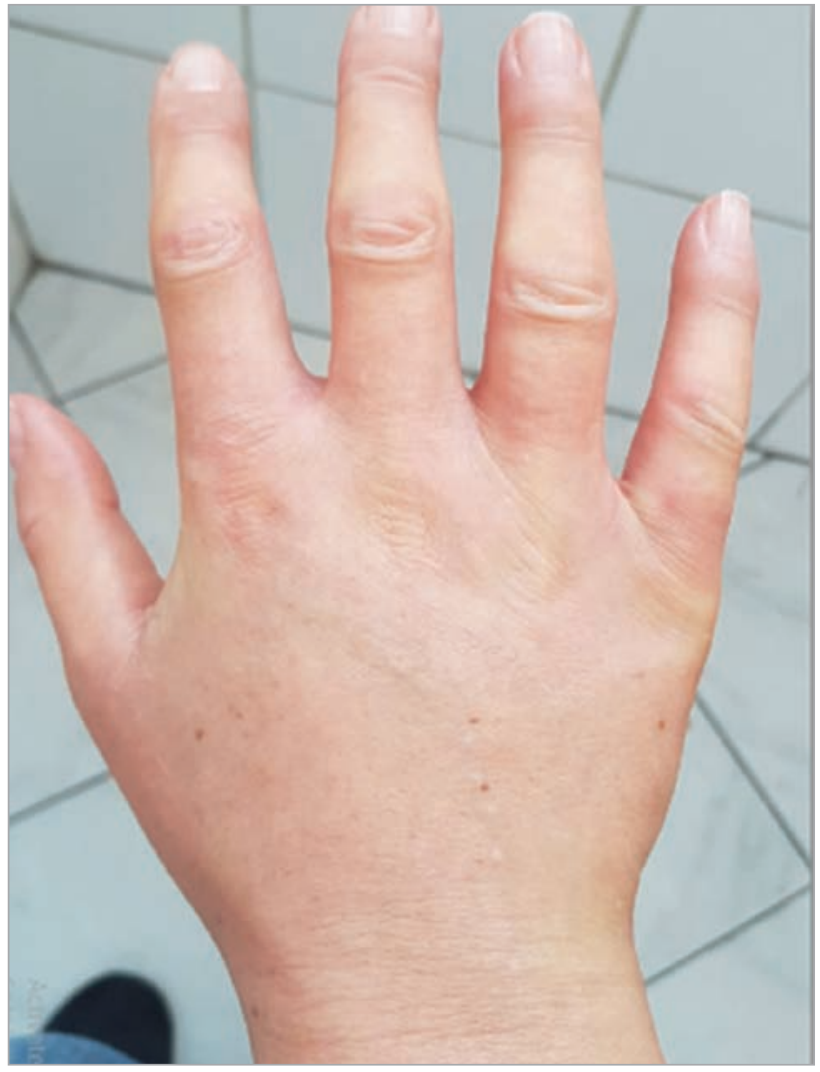

FIGURE 4. Accentuated sclerodactility with movement impairement

or to modern angiotensin-converting enzyme inhibitor therapy, the condition was universally fatal within a few weeks from onset, yet despite great strides being made in managing this complication, five-year mortality rate remains significant and can reach up to $35-40 \%$ (4). It is notable that although ACE inhibitor therapy is the first-line treatment in scleroderma renal crisis, there is currently no evidence to support its use as a prophylactic measure (5).

Up to $20 \%$ of cases can occur before the diagnosis of systemic sclerosis is formally established, with little to no skin involvement observed during clinical examination (6). A high index of suspicion is therefore necessary in any patient, particularly female, presenting with progressive acute kidney failure and malignant hypertension, in the absence of other underlying medical conditions.

In 2014, two similar clinical cases were presented by Bhavsar et al., presenting with microangiopathic hemolytic anemia, thrombocytopenia, and acute kidney injury. After 6 weeks, respectively 9 months since renal onset, patients started developing rapidly-progressive diffuse skin thickening and strongly positive anti-RNA polymerase III antibodies. The author suggests that testing for these autoantibodies might help in differentiating from thrombotic thrombocytopenic purpura or hemolytic uremic syndrome (7). 


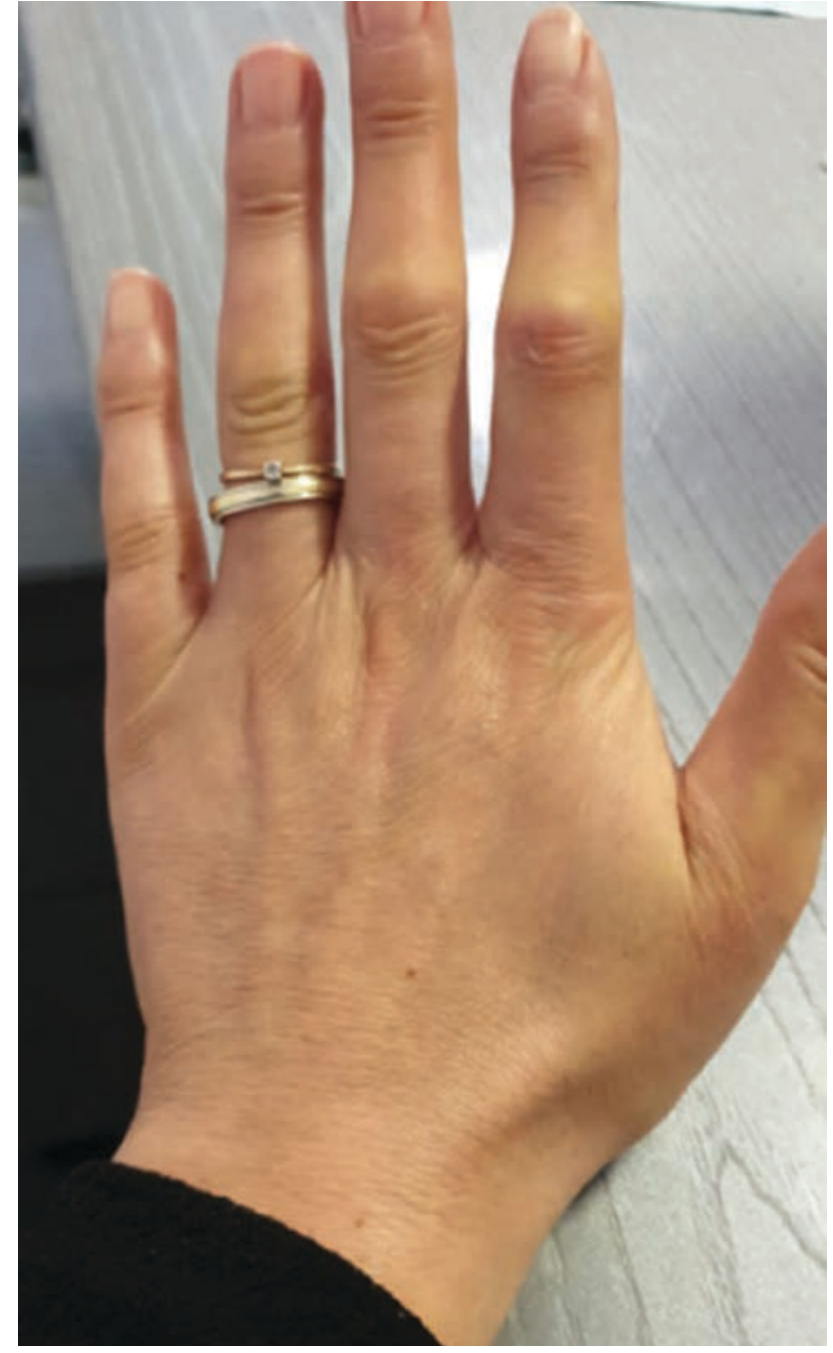

FIGURE 5. Hand aspect after Rituximab treatment showing disappearance of indurated areas

The use of corticosteroids for the management of systemic sclerosis is controversial, as evidence of their beneficial role is limited, while the risk of precipitating renal crisis is significant (8). Therefore, they should be used sparingly and only when absolutely necessary, preferably in the lowest dose possible, for brief intervals of time. Their benefit is mainly limited to improving aching and stiffness in the early stages of the disease, with no long-term impact on skin and organ involvement (1).

Anti-RNA polymerase III antibody (ARA) positivity is an additional risk factor in developing scleroderma renal crisis and is more often associated with the diffuse cutaneous SSc (7). Furthermore, there is evidence to suggest that ARA positivity correlates with more frequent requirement of dialysis and longer recovery times. However, long-term prognosis seems to not be negatively affected, as ARA positive patients appeared to have a better survival outcome compared with the ARA negative group (9).

There is growing evidence of clinical benefit supporting the use of Rituximab in scleroderma patients, particularly in those with more severe and rapidly progressive forms of disease, refractory to standard immunosuppressive therapy (10). It appears to be especially efficient in managing skin fibrosis and preventing the worsening of lung fibrosis, as was the case with the above presented patient, who showed rapid and significant improvement of cutaneous manifestations (11).

Even since 2014, the EUSTAR group evaluated the efficacy and safety of RTX on a cohort of 63 patients with SSc and showed that mRSS was significantly reduced at follow-up thant baseline (26.6 vs. 20.3), with no significant safety concerns. Moreover, RTX prevented the FVC decline compared to control-group (11).

A very recently published systematic review on effects of RTX in SSc included ten controlled trials that confirmed a statistically significant improvement in forced vital capacity at follow-up, while eight studies showed significant improvements in the skin, evaluated with the modified Rodnan skin score (12).

Notable features of the presented case include the onset of disease occurring one month postpartum, raising difficulties in differential diagnosis due to initial suspicion of preeclampsia or postpartum cardiomyopathy. Patient's renal involvement was the sole presenting feature of systemic sclerosis, with no obvious skin changes, negative autoantibodies and normal nailfold capillaroscopy at onset.

Moreover, the five-year interval free of disease manifestations is particular, with the sole exception of intermittent Raynaud's phenomenon even with lack of treatment.

The rapid and favorable response to anti-CD20 emphasizes its benefits on skin involvement in SSc.

\section{CONCLUSIONS}

Scleroderma renal crisis is a serious, life-threatening complication of systemic sclerosis, requiring early recognition and treatment in order to ensure the best possible outcome. It often occurs early in the course of the disease and can sometimes be the sole presenting feature, preceding cutaneous involvement. ACE inhibitor is the mainstay of SRC if promptly initiated, yet the concern of long term morbidity and mortality still remains. Scleroderma patients should be closely monitored for early signs of organ involvement and treatment should be adapted accordingly. In cases where standard immunosuppressive therapy proves inefficient, Rituximab should be considered as a promising option. 


\section{REFERENCES}

1. Fauci AS. Langford CA. Harrison's Rheumatology. 4th ed. McGraw Hill Education; 2017.

2. Ionescu R. Reumatologie - Curs universitar. Editura Universitara "Carol Davila“; 2017.

3. Sterling G, West JK. Rheumatology Secrets. 4th ed. Elsevier; 2020.

4. Cozzi F, Marson P, Cardarelli S, Favaro M, Tison T, Tonello M, Pigatto E, De Silvestro G, Punzi L, Doria A. Prognosis of scleroderma renal crisis: a long-term observational study. Nephrol Dial Transplant. 2012 Dec;27(12):4398-403.

5. Kowal-Bielecka O, Fransen J, Avouac J, Becker M, Kulak A, Allanore Y, Distler O, Clements P, Cutolo M, et al.; EUSTAR Coauthors. Update of EULAR recommendations for the treatment of systemic sclerosis. Ann Rheum Dis. 2017 Aug;76(8):1327-1339.

6. Logee KM, Lakshminarayanan S. Scleroderma renal crisis as an initial presentation of systemic sclerosis: a case report and review of the literature. Clin Exp Rheumatol. 2015 Jul-Aug;33(4 Suppl 91):S171-4.

7. Bhavsar SV, Carmona R. Anti-RNA polymerase III antibodies in the diagnosis of scleroderma renal crisis in the absence of skin disease. J Clin Rheumatol. 2014 Oct;20(7):379-82.

Conflict of interest: none declared Financial support: none declared
8. Iudici M, van der Goes MC, Valentini G, Bijlsma JW. Glucocorticoids in systemic sclerosis: weighing the benefits and risks - a systematic review. Clin Exp Rheumatol. 2013 Mar-Apr;31(2 Suppl 76):157-65.

9. Bernadette M. Lynch, Henry Penn, Jennifer Harvey, Aine Burns CPD. The Prognosis of Scleroderma Renal Crisis in RNAPolymerase III Antibody-Positive Compared to Rna-Polymerase III Antibody-Negative Patients. Rheumatology. 2014;53(suppl_1):i179.

10. Vilela VS, Maretti GB, Gama LM, Costa CH, Rufino RL LR. Rituximab for the therapy of systemic sclerosis: a series of 10 cases in a single center. Rev Bras Reumatol English Ed. 2016;56(5):458463.

11. Jordan S, Distler JH, Maurer B, Huscher D, van Laar JM, Allanore Y DOER study group. Effects and safety of rituximab in systemic sclerosis: an analysis from the European Scleroderma Trial and Research (EUSTAR) group. Ann Rheum Dis. 2015;74(6):1188-1194.

12. de Figueiredo Caldas MMV, de Azevedo KPM, de França Nunes AC, de Oliveira VH, Pimenta IDSF, de Araújo IDT, Neto FAB, da Silveira Gonçalves de Oliveira AK, Piuvezam G. Is rituximab effective for systemic sclerosis? A systematic review and metaanalysis. Adv Rheumatol. 2021 Feb 27;61(1):15. 